\title{
ESTRATÉGIA SUPRAMOLECULAR PARA A NANOTECNOLOGIA
}

\author{
Koiti Araki \\ Instituto de Química, Universidade de São Paulo, CP 26077, 05513-970 São Paulo - SP, Brasil
}

\begin{abstract}
SUPRAMOLECULAR APPROACH TO NANOTECHNOLOGY. Brazilian science is evolving rapidly and steadly in the last 10 years, reaching the $15^{\circ}$ place in the international ranking. Research in nanotechnology is following a similar way generating new scientific and technological knowledge in several frontiers but specially in the interfaces of two or more areas, where Chemistry is consolidating itself as a central science. In this context, the supramolecular approach is a very promissing one because it allows the build-up of a chemical inteligence using all the sistematized knowledge for the design and development of new nanomaterials and products. The great challenge of Chemistry is not decrease the dimensionality of the materials but instead find ways to increase the dimensionality and structural complexity keeping strict control on the interactions between the components, in order to generate materials with new properties and functionalities. Unfortunately, the current vigorous advancement of scientific research has not been followed by the transformation of such knowhow into patents and produts. Therefore much efforts should be devoted to build a national science and technology program, joining all the segments of the society involved in the technological development (university, institutes of technological research, industry and government) in order to promote the furtherance of the Brazilian technological base. Only in this way it is possible to evolve to a technological society capable to transform the scientific knowledge into wealthy, thus sustaining the socioeconomic development of the country.
\end{abstract}

Keywords: nanotechnology; supramolecular approach; nanomaterials.

\section{INTRODUÇ̃̃O}

Ciência e tecnologia sempre andam lado a lado e dependem do desenvolvimento técnico dos instrumentos de medida, de observação, de análise e de produção, que são utilizados de maneira integrada para desenvolver novos produtos. Assim, os estudos da física e da biologia baseavam-se em materiais de dimensões macroscópicas. Porém, o aperfeiçoamento e desenvolvimento constante da instrumentação, principalmente a partir de meados do século passado, tornaram possível o estudo dos materiais em escalas cada vez menores, chegando à escala nano (Figura 1). Essa estratégia possibilitou a diminuição progressiva dos dispositivos de estado sólido, provocando um aumento extraordinário do número de dispositivos que podem ser acomodados numa mesma área. Outra consequiência foi a diminuição do consumo de energia e a possibilidade de integração de um número sempre crescente de dispositivos, tal que a capacidade dos processadores dobrou a cada 24 meses (Lei de Moore) nos últimos 40 anos. Atualmente um processador de última geração como o Intel Itanium de duplo processador contém mais de $1 \times 10^{9}$ transistores (http://www.intel.com/technology/mooreslaw/index.htm).

Comportamento similar pode ser observado no caso da biologia, que iniciou com a sistematização dos organismos vivos pelos seus fenótipos e atualmente estuda a base molecular dos processos biológicos e de sua expressão. Por outro lado, a Química tem como um dos seus objetivos o estudo dos átomos e suas ligações para formar moléculas, que geralmente são menores que cerca de $1 \mathrm{~nm}$. Por sua natureza intrínseca vem trilhando um caminho bem diferente, pois nesse caso a dificuldade se encontra no controle das interações atômico/moleculares de modo a se gerar sistemas maiores e mais complexos de maneira reprodutível. Ou seja, enquanto nas demais áreas a preocupação foi sempre no sentido de diminuir a dimensionalidade para se atingir a escala nanométrica, no caso da Química a questão que se apresenta é diametralmente oposta, ou seja, como aumentar a dimensionalidade (número de componentes moleculares) e a complexidade estrutural, controlando as interações

\footnotetext{
*e-mail: koiaraki@iq.usp.br
}

entre os diversos constituintes, de modo a gerar novas propriedades e funcionalidades. De fato, a complexidade desses sistemas pode ser comparável à dos sistemas biológicos. Assim, os conhecimentos desenvolvidos até o momento ainda não são suficientes para se produzir em grande escala materiais de composições mais complexas, com alta organização atômica/molecular. Vários exemplos podem ser encontrados, mas o caminho trilhado pela química inorgânica é emblemático. A Química Inorgânica tradicional foi desenvolvida até meados do século passado, sendo os conhecimentos sobre as ligações, reatividade, estruturas e propriedades dos materiais e compostos simples, de praticamente todos os elementos da tabela periódica, extensamente estudados e sistematizados. Os desafios encontravam-se justamente na preparação de novos materiais que apresentassem propriedades ou funcionalidades diferenciadas. Uma das vertentes foi o desenvolvimento da Química Bioinorgânica, que visava a utilização dos sistemas biológicos como modelos para se tentar compreender a química associada aos diversos elementos essenciais. Apesar dessa química utilizar compostos muitas vezes bastante familiares, logo se percebeu que as propriedades eram significativamente diferentes, sendo associadas a efeitos eletrônicos e estruturais. Porém, mesmo a preparação dos sistemas biomiméticos mais simples mostraram-se bastante complexas, pois as geometrias, distâncias e ângulos de ligação eram geralmente distintas daquelas convencionais. De fato, a preparação de sistemas biomiméticos ainda continua sendo um desafio formidável. É necessário desenvolver estratégias adequadas, que possibilitem a utilização do conhecimento químico sistematizado para a elaboração e controle dos processos de preparação.

Apesar das dificuldades, atualmente vários produtos nanotecnológicos já se encontram no mercado. Os pneus são fabricados há muito tempo com compósitos de borrachas com negro de fumo. Mais recentemente raquetes de tênis começaram a ser fabricadas com compósitos contendo nanotubos de carbono. Os produtos de beleza e farmacêuticos contêm nanopartículas e materiais micro e nanoencapsulados de modo a melhorar características tais como biodisponibilidade, controle da cinética de liberação e estabilidade. Os revestimentos podem ser nanoestruturados de modo a conferir 
propriedade auto-limpante à superfície de tecidos e pinturas pela ação do efeito lotus ${ }^{1-8}$. Filmes de $\mathrm{TiO}_{2}$ estão sendo utilizados para conferir propriedades auto-limpantes quando irradiados com luz solar. Nanopartículas de prata estão sendo incorporadas a vários produtos poliméricos, têxteis, resinas, cerâmicas etc para conferir propriedades anti-bactericidas ${ }^{9}$, dentre muitos outros. A maior partes dos produtos se vale de uma tecnologia relativamente simples, não envolvendo múltiplos componentes e interfaces, e controle acurado da dinâmica dos eventos e processos nos domínios do espaço, tempo e energia. Um exemplo real de produto com estas características são os microprocessadores acima citados, envolvendo um número extraordinariamente grande de dispositivos, mas ainda usando a junção de poucos materiais de estado sólido.

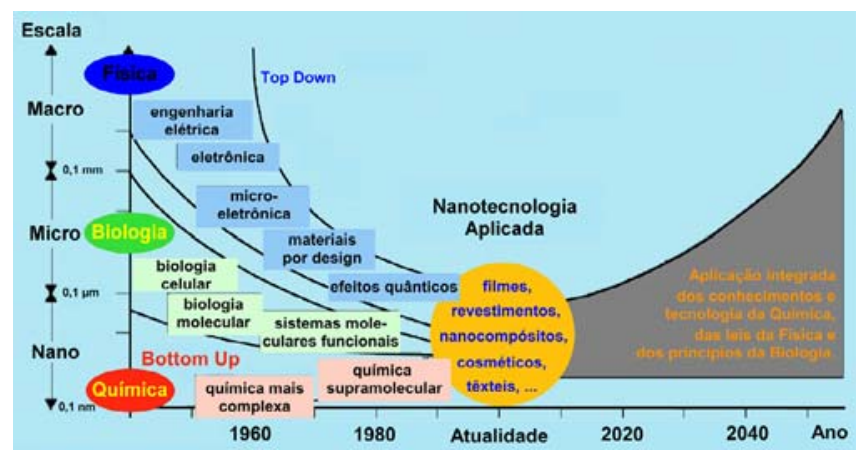

Figura 1. Esquema mostrando a evolução da ciência dos materiais (baseado nos estudos da Bachmann/Hörber), a convergência das três principais ciências naturais para gerar a nanotecnologia atual e integração crescente no futuro, gerando número crescente de produtos

\section{NANOTECNOLOGIA NO BRASIL}

A constante busca por novos conhecimentos e a necessidade de dominar novas tecnologias, fundamentais para enfrentar a acirrada competição num mercado globalizado, estão rapidamente impelindo a humanidade para a próxima revolução tecnológica. Esta deve se basear no domínio dos materiais em escala nano, ou seja, a região entre o universo dos átomos e das moléculas e o universo que denominamos de macroscópico (maior que cerca de $1 \mu \mathrm{m}$ ). É nessa faixa que aparecem as propriedades mesoscópicas dos materiais, como o confinamento quântico, a condução por tunelamento ressonante, além de novas propriedades catalíticas, magnéticas, ópticas, topológicas etc que eventualmente serão exploradas no desenvolvimento de tecnologias inovadoras. Nesse contexto estão sendo preparados diversos tipos de nanomateriais como nanopartículas ${ }^{10,11}$, nanofios ${ }^{12-17}$, nanocompósitos, materiais nanoestruturados, nanoligas, nanotubos ${ }^{18-27}$ etc, que devem provocar um grande impacto científico e tecnológico, principalmente tendo em vista suas excepcionais propriedades elétricas, mecânicas, ópticas, magnéticas e de transporte. Por exemplo, é de amplo conhecimento que os nanotubos de carbono apresentam excepcionais propriedades elétricas e mecânicas, abrindo novas possibilidades de aplicações nas mais diversas áreas de pesquisa e desenvolvimento da atualidade.

Um levantamento no banco de dados Web of Science do ISI, utilizando a expressão chave "nanotechnology or nanocomposite or nanoparticle or nanomaterial or nanotube or supramolecular" e como endereço "Brazil and quim" para realizar a busca, mostra que há um crescente engajamento dos pesquisadores brasileiros nesse importante e atual campo de pesquisa. O levantamento foi realizado de forma abrangente no ISI, de tal modo que todas as contribuições contendo aquelas palavras-chave no título ou no "abstract" foram consideradas. Apesar de não pretender ser completo (desde já peço desculpas pelas omissões não intencionais), é um bom indicativo das atividades de pesquisa realizadas no país naquelas áreas. Por exemplo, pode-se verificar na Figura 2 que as contribuições se iniciaram na década de $90 \mathrm{com}$ algumas publicações por ano, sendo que esse número atingiu cerca de 10 no final da mesma década. Contudo, o número aumentou para mais de 40 nos anos subseqüentes, atingindo um novo patamar a partir de 2000 , seguindo a tendência mundial. O número de citações dos respectivos artigos segue um comportamento similar mantendo-se praticamente constante na década de 90 mas aumentando exponencialmente a partir de 2000 , totalizando cerca de 370 contribuições até o presente. De fato, pode-se projetar o acúmulo de cerca de 1000 citações para o final de 2007, mostrando o impacto crescente da produção intelectual brasileira em nanociência e nanotecnologia pela comunidade química.
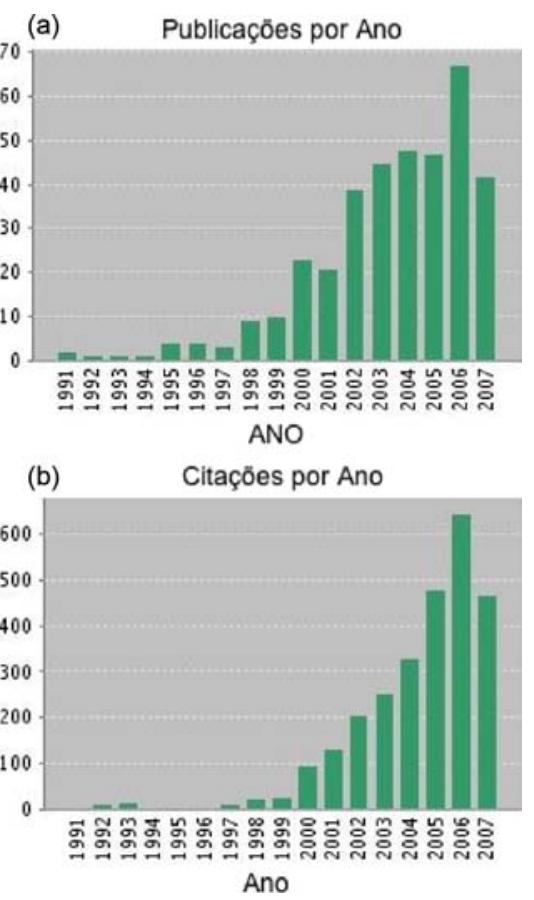

Figura 2. a) Gráficos mostrando o número de publicações e b) o número de citações recebidas pelo mesmo conjunto de publicações por ano, obtidos no portal http://portal.isiknowledge.com utilizando a expressão chave "nanotechnology or nanocomposite or nanoparticle or nanomaterial or supramolecular" e como endereço "Brazil and quim"

\section{Principais linhas e contribuições}

A comunidade química brasileira tem acompanhado a tendência mundial aumentando sua produção intelectual em nanociência e nanotecnologia, principalmente nas universidades das regiões sudeste e sul, no centro-oeste principalmente na Universidade de Brasília e no nordeste principalmente na Universidade Federal de Pernambuco. As linhas de pesquisa são as mais variadas e têm alcançado índices de impacto muito expressivos. Dentre os artigos mais citados no levantamento realizado no ISI, foram encontrados principalmente estudos nas áreas de nanomateriais magnéticos ${ }^{28,29}$, química e nanomateriais supramoleculares ${ }^{30-37}$, nanocompósitos de polímeros e óxidos de metais de transição (principalmente nanopartículas e óxidos lamelares ${ }^{38-42}$, nanoencapsulamento ${ }^{43}$, nanotubos de carbono ${ }^{44,45}$; filmes, interfaces e dispositivos ${ }^{34,35,37,46,47}$ e células solares ${ }^{48-50}$. Essas contribuições são brevemente comentadas abaixo. 
Vaz e colaboradores ${ }^{28}$ exploraram a físico-química de uma família de magnetos moleculares duros e macios contendo três carreadores de spin (complexos de $\mathrm{Cu}$ (II), $\mathrm{Co}$ (II) e $\mathrm{Mn}$ (II)), ou seja, [(Etrad)(2)Mn$2\{\mathrm{Cu}($ opba $)\}(3)(\mathrm{DMSO})(0,5)] \cdot 0,25 \mathrm{H}(2) \mathrm{O}(2)$ e $[($ Etrad $)(2) \mathrm{Co}-$ $2\{\mathrm{Cu}(\mathrm{opba})\}(3)(\mathrm{DMSO})(1,5)] \cdot 0,25 \mathrm{H}(2) \mathrm{O}(3)$, onde $\operatorname{Etrad}(+)$ é o radical cátion 2-(1-etilpiridínio-4-il)-4,4,5,5-tetrametilimidazolina-1oxil-3-óxido, e opba é orto-fenileno-bis(oxamato), explorando principalmente as propriedades coercitivas e o efeito memória daqueles materiais. Por outro lado, Morais e colaboradores ${ }^{29}$ prepararam ferrofluidos iônicos baseados em nanopartículas de ferritas de cobalto com distribuição de tamanho estreita, obtidas controlando-se a velocidade de agitação e os processos de difusão. Os processos de peptização e estabilização também foram estudados e o material caracterizado por TEM e espectroscopia Mössbauer.

Vários grupos atuam em nanomateriais e química supramolecular, destacando-se os Grupos do IQ-USP, UFPE e URGS. A química supramolecular baseada na química de coordenação vem sendo desenvolvida por grupos do Instituto de Química - USP, visando principalmente a obtenção de supermoléculas e sistemas supramoleculares baseados em complexos de rutênio, porfirinas, porfirazinas e compostos análogos ${ }^{34,35,37}$ e dendrímeros ${ }^{31,51}$, além da utilização dos mesmos para a preparação de catalisadores multi-eletrônicos, interfaces e dispositivos moleculares, principalmente sensores químicos e liberadores foto-induzidos de fármacos e outras espécies ${ }^{31}$ e fotossensibilizadores para terapia fotodinâmica ${ }^{33}$. Por outro lado, Sá e colaboradores ${ }^{30}$ publicaram um extenso review sobre dispositivos moleculares de conversão de luz baseados em complexos de lantanídios, focando particularmente os trabalhos realizados por seu grupo na UFPE, baseados numa estratégia teórica-experimental. A química supramolecular de líquidos iônicos vem sendo sistematicamente desenvolvida na UFRGS. Por exemplo, foi realizado o estudo das estruturas supramoleculares formadas pelas interações entre os cátions 1,3-dialquilimidazólio e ânions não-coordenantes, tais como tetrafluoroborato, hexafluorofosfato e tetrafenilborato ${ }^{32}$, utilizando técnicas tais como difração de raios-X, espectroscopia $\mathrm{RMN}^{1} \mathrm{H}$, condutividade e microcalorimetria. Tais estudos mostraram que esses materiais no estado sólido são melhor descritos como sendo polímeros supramoleculares conectados por ligações de hidrogênio entre os cátions imidazólio e os respectivos ânions.

Vários grupos também vêm atuando na área de nanocompósitos de polímeros e óxidos de metais de transição, mas um volume considerável de estudos foi realizado no sentido de se preparar materiais condutores ou semicondutores utilizando $\mathrm{V}_{2} \mathrm{O}_{5}$ como matriz hospedeira de polipirrol e polianilina. Esses materiais foram caracterizados por métodos eletroquímicos, TG e DSC, espectroscopia Raman e microscopia eletrônica de varredura, mostrando que polianilina é oxidada nos compósitos aumentando a condutividade elétrica ${ }^{40,41}$. Material análogo foi preparado pela técnica camada por camada de modo a maximizar a interação entre polianilina e $\mathrm{V}_{2} \mathrm{O}_{5}$, promovendo efeitos cooperativos que aumentaram a capacidade de carga do material na forma de fil$\mathrm{me}^{42}$. Nanocompósitos do hidróxido duplo lamelar $\mathrm{Al}_{0.33} \mathrm{Mg}_{0.67}(\mathrm{OH})_{2}\left(\mathrm{C}_{12} \mathrm{H}_{25} \mathrm{SO}_{4}\right)_{0.33} \cdot 0,64 \mathrm{H}_{2} \mathrm{O}$ com poli-óxido de etileno foram obtidos e caracterizados por diversas técnicas indicando que o material apresenta estrutura ordenada, e dodecilsulfato intercalado no espaço interlamelar ${ }^{38}$. Por outro lado, também foram preparados materiais híbridos orgânico/inorgânicos contendo dióxidos de titânio e de estanho e polianilina pelo método sol-gel. Estudos foram realizados por análise térmica, difratometria de raios-X, espectroscopias UV-Vis, Raman e infravermelho e eletroquímica, bem como microscopia eletrônica de transmissão, evidenciando a formação de nanopartículas ou nanotubos dos óxidos e polianilina na forma emeraldina, podendo formar estruturas do tipo casca-caroço quando a quantidade de polianilina é equivalente à cerca de $10 \%$ em massa ${ }^{39}$.
Outro material de grande importância, com um Instituto do Milênio dedicado exclusivamente para o seu estudo, são os nanotubos de carbono. As propriedades condutoras e espectroscópicas, além da extraordinária resistência mecânica, são os temas mais abordados, juntamente com aplicações em dispositivos como sensores químicos e supercapacitores, ou como nanorrecipientes para diversas espécies. Corio e colaboradores ${ }^{44,45}$ estudaram o espalhamento Raman de superfície de filmes de nanotubos de carbono "single wall" sobre eletrodos de platina, mostrando que há uma variação das freqüências, intensidades e perfil das bandas em função do potencial aplicado. Tais variações foram atribuídas ao preenchimento dos diferentes estados eletrônicos dos nanotubos metálicos e semicondutores. Além disso, foi mostrado que a inserção de espécies doadoras $\left(\mathrm{Ag}^{0}\right)$ e receptoras de elétrons $\left(\mathrm{CrO}_{3}\right)$ altera o nível de Fermi dos nanotubos e, conseqüentemente, as propriedades eletrônicas e vibracionais dos mesmos.

Diversos grupos de pesquisa na área de química vêm desenvolvendo aplicações de filmes finos em interfaces e dispositivos, tais como sensores químicos, dispositivos eletroluminescentes, células fotoeletroquímicas, dispositivos eletrônicos tais como memórias e transistores moleculares, dentre outros. Tiveram impacto significativo os trabalhos realizados pelo grupo de Toma e Araki ${ }^{34,35,37,46,47}$ envolvendo a aplicação de filmes de meso-tetra(piridil)porfirinas coordenadas a complexos de rutênio polipiridinas ou clusters trigonais de acetato de rutênio como materiais ativos de sensores de espécies tais como sulfito, nitrito, ácido ascórbico e oxigênio, bem como os trabalhos relacionados com células fotoeletroquímicas de Grätzel. Kleverlaan e colaboradores ${ }^{48}$ estudaram as propriedades fotofísicas de complexos binucleares de rutênio e ródio bipiridina em solução, bem como adsorvido na superfície de um filme nanocristalino de $\mathrm{TiO}_{2}$. O estudo do processo de injeção de elétrons mostrou que cerca de $1 / 3$ das espécies no estado excitado realizam diretamente esse processo, mas as demais injetam elétrons somente após uma reação de transferência de elétrons intramolecular envolvendo a formação da espécie Rh(II)-Ru(III). Por outro lado, Garcia e colaboradores ${ }^{49,50}$ realizaram estudos voltados para o desenvolvimento de células fotoeletroquímicas utilizando diversas moléculas como sensibilizadores, inclusive pigmentos naturais.

Dentre as várias tecnologias empregadas na geração de nanoprodutos, talvez a de micro e nano-encapsulamento seja uma das mais interessantes, pois permite gerar os análogos das nanopartículas utilizando materiais líquidos ou sólidos. Além disso, o controle da composição e características da camada protetora, ou o tipo de cápsula gerada (matricial, por coacervação, polimérica) possibilita o controle da cinética de liberação do(s) componente(s) encapsulado(s) nas mais diversas condições, além de poder promover a estabilização dos materiais ativos. A versatilidade e funcionalidade dos materiais assim gerados está sendo empregada nos mais diversos segmentos, destacando-se os setores farmacêutico, de alimentos e de cosméticos. Esse tipo de tecnologia vem sendo explorada por Pohlmann e colaboradores ${ }^{43}$, por exemplo, para o desenvolvimento de processos industriais para a produção de suspensões de nanopartículas poliméricas, que podem ser utilizadas como sistemas para entrega de drogas. Assim, suspensões de nanocápsulas e nanoesferas foram preparadas por nanoprecipitação de poliéster, usando $\mathrm{SiO}_{2}$ como coadjuvante, pela técnica de "spray-drying".

\section{ESTRATÉGIA SUPRAMOLECULAR PARA A NANOTECNOLOGIA}

A Ciência Química evoluiu dramaticamente nas últimas décadas e vem assumindo um papel cada vez mais importante no contexto global, particularmente no desenvolvimento das nanociências 
e da nanotecnologia pela estratégia "bottom-up". Essa estratégia é inerentemente química, pois se baseia na preparação de nanomateriais e nanossistemas a partir dos átomos e moléculas constituintes. Porém, para tal é necessário dominarmos os princípios físicoquímicos envolvidos não somente na formação de ligações covalentes ou iônicas fortes. Também é necessário compreendermos a dinâmica e a energética dos fenômenos regidos por interações fracas e o efeito da organização molecular por elas induzidas sobre as propriedades dos materiais. Por outro lado, podemos simplesmente focalizar nas propriedades intrínsecas de um dado material puro mas reduzido à escala nano, como as propriedades bactericidas da prata na forma de nanopartículas ${ }^{9}$, ou a condutividade elétrica, a resistência mecânica e as propriedades de incorporação de substâncias dos nanotubos de carbono, ou as propriedades magnéticas de nanopartículas de $\mathrm{Fe}_{2} \mathrm{O}_{3}$, ou as propriedades luminescentes de nanopartículas de CdS. Contudo, propriedades mais interessantes e quimicamente controláveis são obtidas quando ocorre a interação entre diferentes materiais orgânicos e/ou inorgânicos gerando uma ou mais interfaces ou junções, justamente o escopo da Química Supramolecular ${ }^{52-55}$.

A Química Supramolecular está baseada na organização atômi$\mathrm{ca} /$ molecular e na interação sinérgica entre os constituintes de sistemas multi-componentes estruturalmente mais ou menos bem definidos, visando a obtenção de novas funcionalidades e propriedades. Nesse sentido, supermoléculas com propriedades específicas têm sido projetadas, tais como auto-montagem, reconhecimento molecular, transformação, transporte e sinalização. A organização molecular também tem sido explorada para promover a transferência vetorial de elétrons (ou separação de cargas fotoinduzida) e de energia (ou efeito antena), fenômenos essenciais nos processos de conversão da luz solar em energia química ou elétrica ${ }^{56,57}$. Além disso, as supermoléculas podem ser concebidas de modo a serem inteligentes, isto é, com capacidade de compreender e responder a sinais químicos sendo, portanto, capazes de executar certas funções quando combinados com outros sistemas ou materiais de maneira adequada. Ou seja, em função da organização molecular é possível conceber sítios de reconhecimento molecular e possibilitar processos de sinalização, comunicação e transformação molecular, além dos fenômenos de auto-montagem, auto-replicação e auto-reparação. Além disso, pode-se organizar os sítios ativos de modo a possibilitar processos dinâmicos com controle dos eventos nas dimensões de espaço, tempo e energia. Tal controle pode tornar possível processos de conversão de energia como na fotossíntese, além do transporte, armazenamento e processamento de informações. O desenvolvimento de espécies moleculares funcionais também poderá levar a dispositivos baseados em sistemas químicos integrados ${ }^{58}$, por exemplo, nas áreas de dispositivos moleculares, da eletrônica e fotônica molecular ${ }^{56,57}$, bem como da nanotecnologia. Assim, fases ou sólidos constituídos por um grande número de moléculas ou supermoléculas, formando superestruturas tridimensionais mais ou menos ordenadas, contendo sítios de reconhecimento molecular e sítios catalítica, eletrocatalítica e/ou fotoquímicamente ativos podem ser concebidos e realizados. Filmes finos orgânicos, inorgânicos, poliméricos e/ou híbridos, materiais lamelares, nanopartículas e outros nanomateriais, bem como micelas, vesículas e membranas podem ser utilizadas para tal finalidade.

A "estratégia supramolecular" pode ser utilizada em quaisquer situações em que se queira modificar a funcionalidade de compostos e de materiais por combinação com outros materiais ou modificação química de sua superfície e interfaces. Além disso, apresenta a vantagem de se poder utilizar os conhecimentos químicos sistematizados já existentes para a montagem dos nanomateriais supramoleculares. Ou seja, há a possibilidade de se tentar montar sistemas mais complexos com as propriedades desejadas selecionando adequadamente os componentes individuais. De fato dois tipos de componentes são necessários: os ativos e os estruturais e/ ou conectores. Os componentes ativos são aqueles responsáveis pela realização das etapas que levam à execução de um dado processo, ou seja, de reconhecimento molecular, de ligação/orientação/ativação, de absorção ou emissão de luz, de transferência de elétrons ou de energia etc. Por outro lado, os componentes estruturais são necessários para se organizar os componentes ativos no espaço, de modo a criar sítios que promovam a aproximação dos substratos com a orientação e a energia adequadas para a realização das transformações desejadas. Por exemplo, os componentes podem ser selecionados de modo a gerar gradientes de potenciais redox ou de níveis de energia, fazendo com que os processos de transferência de elétrons ou de energia ocorram preferencialmente numa dada direção (transferência vetorial).

\section{Porfirinas tetraclusters como catalisadores multieletrônicos}

As porfirinas tetracluster são exemplos interessantes de como se pode alterar as propriedades de uma dada molécula por efeitos eletrônicos de outras moléculas, no caso complexos trinucleares de acetato de rutênio. Abaixo serão descritas suscintamente algumas de suas características. As supermoléculas, designadas M-TCP (Figura 3), possuem quatro grupos $\left[\mathrm{Ru}_{3} \mathrm{O}(\mathrm{Ac})_{6}(\mathrm{py})_{2}\right]^{+}$ligados às $\mathrm{M}-4-$ TPyP (onde $\mathrm{M}=\mathrm{Mn}^{3+}$ e $\mathrm{Co}^{3+}$ ). A interação entre os íons rutênio no cluster é forte, fazendo com que os potenciais redox dos mesmos estejam distribuídos na faixa de $-3 \mathrm{a}+3 \mathrm{~V}$, separados de mais ou menos $1 \mathrm{~V}$. Os voltamogramas cíclicos ${ }^{37,59}$ geralmente exibem três ondas intensas e reversíveis em torno de $-1,14 ; 0,16$ e 1,23 V, além de uma onda anódica irreversível em $\sim 2,3 \mathrm{~V}$. Estas podem ser atribuidas aos pares $\mathrm{Ru}^{\text {III }} \mathrm{Ru}^{\text {III }} \mathrm{Ru}^{\mathrm{II}} / \mathrm{Ru}^{\text {III }} \mathrm{Ru}^{\text {II }} \mathrm{Ru}^{\text {II }}, \mathrm{Ru}^{\text {III }} \mathrm{Ru}^{\text {III }} \mathrm{Ru}^{\text {III/ } / ~}$

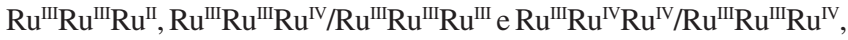
respectivamente, por comparação com compostos análogos e baseado nos resultados de espectroeletroquímica. Além disso, as ondas de menor intensidade podem ser atribuídas aos processos $\mathrm{P}^{2+++} / \mathrm{P}^{+/ 0} /$ $\mathrm{P}^{0 /-} / \mathrm{P}^{-/ 2-}$, respectivamente, além de reações redox associados com o íon de metal de transição coordenado ao anel porfirínico, ou seja $\mathrm{Mn}(\mathrm{III} / \mathrm{II}) \mathrm{P} \mathrm{e} \mathrm{Co(III/III/I)P.}$

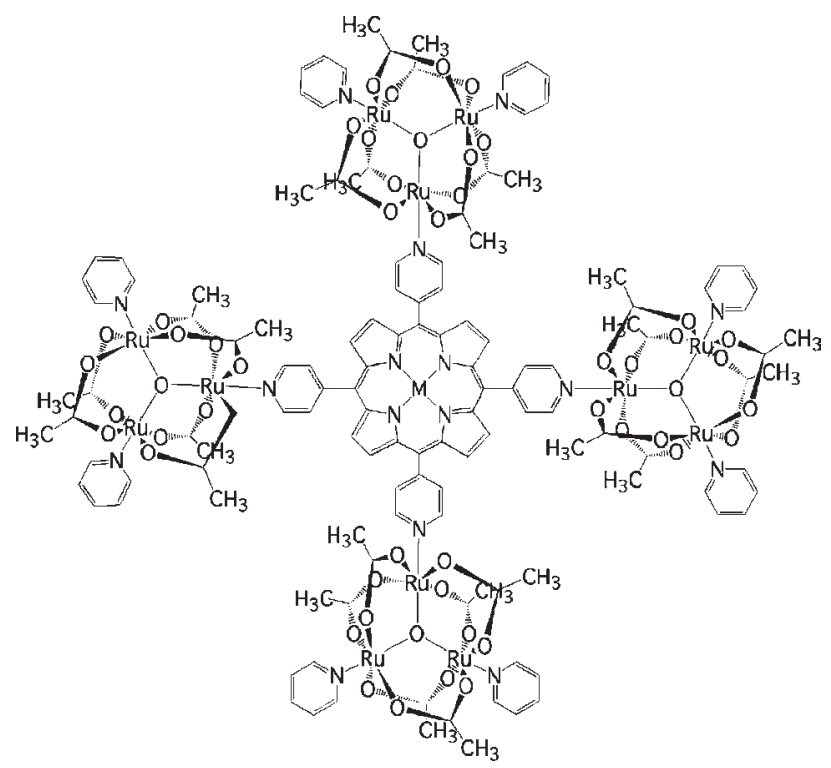

Figura 3. Estrutura da supermolécula tetracluster porfirina, $M-T C P$, onde $M$ $=\mathrm{Mn}^{3+}$ ou $\mathrm{Co}^{3+}$ 
Porfirina cluster como modelo de citocromo-c oxidase

A redução tetraeletrônica de $\mathrm{O}_{2}$ a $\mathrm{H}_{2} \mathrm{O}$ é uma reação fundamental realizada pelos organismos vivos no final da cadeia respiratória, durante o ciclo de produção de energia nas mitocôndrias. Do ponto de vista tecnológico, a realização da mesma reação em condições ambiente ainda continua sendo um dos grandes desafios para o desenvolvimento de células de combustíveis mais eficientes e de menor custo. De fato a redução de $\mathrm{O}_{2}$ diretamente a água é uma reação extremamente difícil, devido ao fato de envolver vários elétrons e estar associada a mecanismos de transporte de prótons. Mas, no final da década de 70, Collman e Anson ${ }^{60-62}$ sintetizaram uma cobalto porfirina cofacial capaz de promover tal reação, quando adsorvida em eletrodos de carbono pirolítico. Estudos detalhados variando-se a distância entre os planos dos anéis porfirínicos e os metais coordenados aos mesmos mostraram que o mecanismo de redução tetraeletrônica deveria envolver a coordenação simultânea da molécula de $\mathrm{O}_{2}$ a dois sítios metálicos, como no citocromo-c oxidase. Posteriormente, Anson e Shi ${ }^{63-66}$ tiveram sucesso na preparação de eletrodos modificados com o complexo [Co-4-TPyP $\left\{\mathrm{Ru}\left(\mathrm{NH}_{3}\right)_{5}\right\}_{4}$, pela reação de Co-4-TPyP com $\left[\mathrm{Ru}\left(\mathrm{NH}_{3}\right)_{5}\left(\mathrm{H}_{2} \mathrm{O}\right)\right]^{2+}$ in situ, em filmes de Nafion. A supermolécula obtida demonstrou ser um eficiente catalisador para a redução tetraeletrônica de $\mathrm{O}_{2}$, mas somente quando pelo menos três complexos de rutênio estão coodenados àquela cobalto porfirina. Em virtude desses resultados, foi sugerido que a ativação se deve principalmente ao efeito de retrodoação dos complexos de rutênio(II) periféricos e não apenas ao número de elétrons disponíveis no catalisador para promover a reação multi-eletrônica.

As propriedades espectroscópicas, eletroquímicas e eletrocatalíticas do Co-4-TPyP coordenado a quatro clusters $\left[\mathrm{Ru}_{3} \mathrm{O}\right.$ $(\mathrm{OAc})_{6}(\mathrm{py})_{2}$ ], CoTCP $35,37,47,67-69$ foram estudadas pelo grupo de Química Supramolecular do IQ-USP. Os voltamogramas cíclicos de eletrodos de carbono vítreo quimicamente modificados com CoTCP, em solução aerada de $\mathrm{KNO}_{3} 0,50 \mathrm{M}$ e tampão acetato $0,05 \mathrm{M}(\mathrm{pH}=4,7)$, apresentaram uma intensa onda catódica em $E_{\mathrm{pc}} \cong 0,2 \mathrm{~V}$. A corrente de pico é proporcional à raiz quadrada da velocidade de varredura, evidenciando que a corrente está sendo limitada pela difusão do oxigênio dissolvido em solução. Os voltamogramas RDE apresentam um perfil sigmoidal na faixa de 100 a $3000 \mathrm{rpm}$, sugerindo que tanto o transporte de elétrons através do filme quanto a transferência de elétrons do filme para o $\mathrm{O}_{2}$ são rápidos, sendo a etapa limitante o transporte de $\mathrm{O}_{2}$ até a interface filme/solução. Além disso, apenas uma quantidade insignificante de $\mathrm{H}_{2} \mathrm{O}_{2}$ foi detectada no eletrodo de anel de platina polarizado a 1,22 V nos voltamogramas RRDE (Figura 4). Estudos análogos realizados com filmes obtidos por montagem

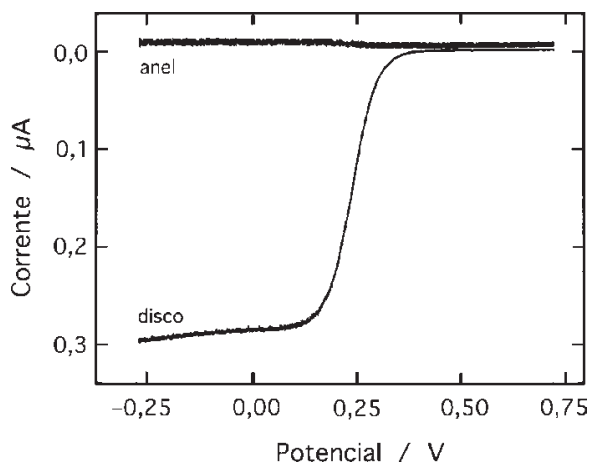

Figura 4. Voltamograma de anel-disco rotatório de um eletrodo de carbono vítreo modificado com filme de CoTCP (velocidade de varredura no disco $=$ $10 \mathrm{mV} \mathrm{s}^{-1}, \omega=1000 \mathrm{rpm}$ ) numa solução de $\mathrm{KNO}_{3} 0,50 \mathrm{M}$, tampão acetato $0,05 \mathrm{M}, \mathrm{pH}=4,7, \mathrm{~T}=25^{\circ} \mathrm{C}$, saturada com ar. $O$ anel de platina foi polarizado a 1,22 V para garantir a oxidação do $\mathrm{H}_{2} \mathrm{O}_{2}$, que eventualmente seja gerado no disco, a $\mathrm{O}_{2}$ camada por camada de um filme de CoTCP/ZnTPPS indicaram que este material também apresenta atividade eletrocatalítica análoga para redução tetraeletrônica de $\mathrm{O}_{2}$. Logo, CoTCP apresenta uma extraordinária atividade eletrocatalítica para redução tetraeletrônica de $\mathrm{O}_{2}$ a $\mathrm{H}_{2} \mathrm{O}$, sendo a ativação da $\mathrm{CoP}$ decorrente de fatores eletrônicos induzidos pelos clusters periféricos.

\section{Porfirina cluster como modelo de citocromo P-450}

Manganês e ferro porfirinas ${ }^{70-72}$ têm sido largamente exploradas como modelos biomiméticos do citocromo P-450, pois são capazes de promover a reação de oxidação de substratos orgânicos por agentes oxidantes doadores de átomos de oxigênio. Porém, porfirinas convencionais como MnTPP são relativamente susceptíveis às reações de oxidação, sendo rapidamente decompostas no meio reacional. Assim, grupos receptores de elétrons como os halogênios foram introduzidos nos anéis pirrólicos e nas fenilas da MnTPP e FeTPP, com o intuito de se aumentar a resistência à oxidação, bem como para aumentar a atividade catalítica. De fato, essas manganês porfirinas substituídas tendem a ser catalisadores mais eficientes e estáveis, em reações de epoxidação e hidroxilação de substratos orgânicos.

Torna-se evidente que uma supermolécula como o MnTCP apresenta grande potencialidade ${ }^{47}$ como modelo de citocromo P-450, não apenas pela presença dos cluster trigonais de acetato de rutênio no estado (III,III,III), que se comportam como grupos receptores fracos de elétrons, mas também por serem eletroquimicamente ativos. É possível controlar o caráter doador ou receptor dos mesmos simplesmente mudando-se o seu estado de oxidação. O estudo comparativo da atividade catalítica da MnTCP e MnTPyP para a reação de epoxidação de cicloexano revelou que o primeiro é um catalisador muito mais eficiente e seletivo, produzindo cicloexanol (45\%) como único produto de oxidação, enquanto MnTPyP gera uma mistura de cicloexanol (27\%) e cicloexanona (13\%).

$\mathrm{O}$ oxo complexo de alta valência, $\mathrm{O}=\mathrm{Mn}^{\mathrm{V}} \mathrm{P}$, provavelmente, é a espécie ativa responsável por essa reação catalítica, porém, é muito difícil detectá-lo, devido à sua elevada reatividade e conseqüente curto tempo de vida. A mistura de MnTCP e uma suspensão de iodosilbenzeno em dicloroetano, numa cela de fluxo, levou à obtenção de dados consistentes com a formação de $\mathrm{O}=\mathrm{Mn}^{\mathrm{IV}} \mathrm{TCP}$, por espectroscopia UV-VIS. Por outro lado, quando o mesmo experimento foi repetido na presença do substrato cicloexano ${ }^{47}$, nenhuma alteração foi observada nos espectros sucessivos da mistura reacional contendo MnTCP. Porém, quando este catalisador supramolecular foi substituído por MnTPyP, nas mesmas condições experimentais, notou-se a formação de $\mathrm{O}=\mathrm{Mn}^{\mathrm{IV}} \mathrm{TPyP}$. Esses resultados indicam claramente que MnTCP é um catalisador muito mais eficiente. Evidenciam também a formação de uma espécie ativa de alta valência, que reage muito rapidamente com o substrato cicloexano regenerando o complexo de partida, não sendo detectado na escala de tempo dos experimentos. Além disso, um pequeno aumento de absorbância observado em torno de $850 \mathrm{~nm}$ indicou a oxidação dos clusters de rutênio periféricos para o estado (III,III,IV), na espécie ativa $\mathrm{O}=\mathrm{Mn}^{\mathrm{V}} \mathrm{TCP}$, estabilizando o anel porfirínico e aumentando a reatividade daquele oxo complexo de alta valência. Por outro lado, quando CoTCP foi utilizado como catalisador para redução tetraeletrônica de $\mathrm{O}_{2}$, os complexos de rutênio periféricos foram reduzidos ao estado (III,III,II), ativando a cobalto porfirina e deslocando o potencial de redução da porfirina para potenciais mais negativos. Logo, também atuam como cofatores armazenando quatro ou mais equivalentes de oxidação ou redução (Figura 5), facilitando a ocorrência de reações catalíticas multi-eletrônicas. Tais características não podem ser encontradas em catalisadores porfirínicos convencionais, evidenciando as vantagens das supermoléculas e sistemas supramoleculares baseados em com- 
Modulação das Propriedades em Função do Estado de Oxidação

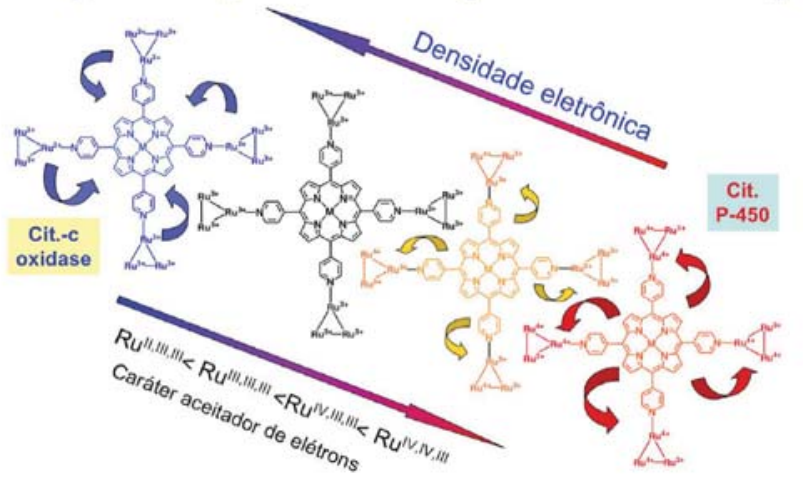

Figura 5. Esquema mostrando o efeito da alteração do estado de oxidação dos complexos de rutênio periféricos e sua ação sobre o sítio catalítido da porfirina. Oxidação transforma-os em receptores fortes e redução em doadores fortes, que por efeitos eletrônicos ativam o sítio cataliticamente ativo, além de atuarem como doadores ou receptores de elétrons

postos de coordenação. Finalmente, vale ressaltar que os catalisadores se ajustam espontaneamente às condições do meio, de modo a maximizar a atividade catalítica tanto para oxidação como para redução, tornando-os "inteligentes" 59 .

\section{CONSIDERAÇÕES FINAIS}

A ciência brasileira está avançando a passos largos, ocupando atualmente a $15^{\text {a }}$ colocação no ranking mundial. As pesquisas em nanotecnologia seguem o mesmo caminho gerando novos conhecimentos científicos e tecnológicos em diversas fronteiras do conhecimento, principalmente nas interfaces entre duas ou mais áreas do conhecimento, aonde a Química vem consolidando um papel cada vez mais central. A estratégia supramolecular é uma das mais promissoras estratégias de atuação dos químicos nessa revolução, pois permite criar uma lógica química para o desenvolvimento de novos nanomateriais e produtos. Atualmente, diversas oportunidades estão à nossa espera principalmente em áreas tais como síntese de nanomateriais em geral, superfícies, interfaces e dispositivos, catalisadores e encapsulamento, aplicáveis nas áreas de energia, meio ambiente, fármacos, cosméticos, alimentos, agronegócios etc. Contudo, é necessário continuar a criar os fundamentos para o desenvolvimento de nanomateriais e sistemas cada vez mais complexos, envolvendo várias interfaces e junções, visando o futuro. Tais estudos devem estar atrelados a uma visão de mercado para que se possa gerar, de fato, produtos. Ou seja, não basta o conhecimento técnicocientífico. É necessário termos políticas de desenvolvimento educacional, científico-tecnológico e industrial integradas, que fomentem a formação de recursos humanos de alta qualidade, atreladas à pesquisa científico-tecnológica e melhoria da infra-estrutura empresarial, legislativa e de inspeção/padronização. Esforços também devem ser canalizados no sentido de se promover mudanças culturais no sentido da valorização da ética e dos conhecimentos técnico-científicos, visando a consolidação de uma sociedade tecnológica. Infelizmente, a performance da comunidade científica brasileira ainda não está sendo acompanhada pela transformação desses conhecimentos em patentes e produtos. Somente quando ocorrer o fechamento desse ciclo virtuoso conectando os diversos segmentos da sociedade (universidades, centros e institutos de pesquisa tecnológica, empresas e governo), estaremos agregando valor aos conhecimentos científicos, transformando-os em riquezas, assim promovendo o desenvolvimento socioeconomico sustentável do País.

\section{AGRADECIMENTOS}

À FAPESP e ao CNPq pelo apoio financeiro e ao Prof. H. E. Toma pela revisão deste trabalho.

\section{REFERÊNCIAS}

1. Guo, Z. G.; Han, F. B.; Wang, L. B.; Liu, W. M.; Thin Solid Films 2007, 515,7190 .

2. Chang, K. C.; Chen, Y. K.; Chen, H.; J. Appl. Polym. Sci. 2007, 105, 1503.

3. Puukilainen, E.; Rasilainen, T.; Suvanto, M.; Pakkanen, T. A.; Langmuir 2007, 23, 7263.

4. Koponen, H. K.; Saarikoski, I.; Korhonen, T.; Paakko, M.; Kuisma, R.; Pakkanen, T. T.; Suvanto, M.; Pakkanen, T. A.; Appl. Surf. Sci. 2007, 253, 5208.

5. Jun, L. A.; Guo, Z. G.; Fang, J.; Hao, J. C.; Chem. Lett. 2007, 36, 416.

6. Chang, K. C.; Chen, H.; Huang, C. K.; Huang, S. I.; J. Appl. Polym. Sci. 2007, 104, 1646.

7. Larmour, I. A.; Bell, S. E. J.; Saunders, G. C.; Angew. Chem., Int. Ed. 2007, 46, 1710 .

8. Hassel, A. W.; Milenkovic, S.; Schurmann, U.; Greve, H.; Zaporojtchenko, V.; Adelung, R.; Faupel, F.; Langmuir 2007, 23, 2091.

9. Gibbs, R. J.; Silver Colloids. Do they work? Newark, 1999.

10. Park, J.; Joo, J.; Kwon, S. G.; Jang, Y.; Hyeon, T.; Angew. Chem., Int. Ed. 2007, 46, 2.

11. Wilcoxon, J. P.; Abrams, B. L.; Chem. Soc. Rev. 2006, 35, 1162.

12. Bruno, M.; Palummo, M.; Ossicini, S.; Del Sole, R.; Surf. Sci. 2007, 601, 2707.

13. Pearton, S. J.; Norton, D. P.; Ren, F.; Small 2007, 3, 1144.

14. Zhu, Q.; Karlsson, K. F.; Pelucchi, E.; Kapon, E.; Nano Lett. 2007, 7, 2227.

15. Knight, M. W.; Grady, N. K.; Bardhan, R.; Hao, F.; Nordlander, P.; Halas, N. J.; Nano Lett. 2007, 7, 2346.

16. Dattoli, E. N.; Wan, Q.; Guo, W.; Chen, Y. B.; Pan, X. Q.; Lu, W.; Nano Lett. 2007, 7, 2463.

17. Sun, J.; Li, J. Q.; Du, X. W.; Lu, Y. W.; Han, X.; Mater. Lett. 2007, 61, 3783 .

18. Gurin, V. A.; Gurin, I. V.; Kolosenko, V. V.; Ksenofontov, V. A.; Mazilova, T. I.; Mikhailovskii, I. M.; Bukolov, A. N.; Technical Physics Letters 2007, $33,534$.

19. Miao, L.; Ina, Y.; Tanemura, S.; Jiang, T.; Tanemura, M.; Kaneko, K.; Toh, S.; Mori, Y.; Surf. Sci. 2007, 601, 2792.

20. Bittencourt, C.; Felten, A.; Douhard, B.; Colomer, J. F.; Van Tendeloo, G.; Drube, W.; Ghijsen, J.; Pireaux, J. J.; Surf. Sci. 2007, 601, 2800.

21. Munoz-Sandoval, E.; Agarwal, V.; Escorcia-Garcia, J.; Ramirez-Gonzalez, D.; Martinez-Mondragon, M. M.; Cruz-Silva, E.; Meneses-Rodriguez, D.; Rodriguez-Manzo, J. A.; Terrones, H.; Terrones, M. Small 2007, 3, 1157.

22. Lee, K. J.; Yoon, S. H.; Jang, J.; Small 2007, 3, 1209.

23. Hang, Q.; Maschmann, M. R.; Fisher, T. S.; Janes, D. B.; Small 2007, 3, 1266.

24. Inami, N.; Mohamed, M. A.; Shikoh, E.; Fujiwara, A.; Sci. Tech. Adv. Mater 2007, 8, 292.

25. Yang, X. M.; Dai, T. Y.; Zhu, Z. X.; Lu, Y.; Polymer 2007, 48, 4021

26. Wu, Y.; Maultzsch, J.; Knoesel, E.; Chandra, B.; Huang, M. Y.; Sfeir, M. Y.; Brus, L. E.; Hone, J.; Heinz, T. F.; Phys. Rev. Lett. 2007, 99.

27. Nyamori, V. O.; Coville, N. J.; Organometallics 2007, 26, 4083.

28. Vaz, M. G. F.; Pinheiro, L. M. M.; Stumpf, H. O.; Alcantara, A. F. C.; Golhen, S.; Ouahab, L.; Cador, O.; Mathoniere, C.; Kahn, O.; Chem. Eur J. 1999, 5, 1486.

29. Morais, P. C.; Garg, V. K.; Oliveira, A. C.; Silva, L. P.; Azevedo, R. B.; Silva, A. M. L.; Lima, E. C. D.; J. Magn. Magn. Mater. 2001, 225, 37.

30. de Sa, G. F.; Malta, O. L.; Donega, C. D.; Simas, A. M.; Longo, R. L.; SantaCruz, P. A.; da Silva, E. F.; Coord. Chem. Rev. 2000, 196, 165.

31. Archut, A.; Vogtle, F.; De Cola, L.; Azzellini, G. C.; Balzani, V.; Ramanujam, P. S.; Berg, R. H.; Chem. Eur. J. 1998, 4, 699.

32. Consorti, C. S.; Suarez, P. A. Z.; de Souza, R. F.; Burrow, R. A.; Farrar, D. H.; Lough, A. J.; Loh, W.; da Silva, L. H. M.; Dupont, J.; J. Phys. Chem. B 2005, 109, 4341 .

33. Onuki, J.; Ribas, A. V.; Medeiros, M. H. G.; Araki, K.; Toma, H. E.; Catalani, L. H.; Di Mascio, P.; Photochem. Photobiol. 1996, 63, 272.

34. Toma, H. E.; Araki, K.; Coord. Chem. Rev. 2000, 196, 307.

35. Araki, K.; Dovidauskas, S.; Winnischofer, H.; Alexiou, A. D. P.; Toma, H. E.; J. Electroanal. Chem. 2001, 498, 152.

36. Haiduc, I.; Zukerman-Schpector, J.; Phosphorus, Sulfur Silicon Relat. Elem. 2001, 171, 171

37. Toma, H. E.; Araki, K.; Alexiou, A. D. P.; Nikolaou, S.; Dovidauskas, S.; Coord. Chem. Rev. 2001, 219, 187. 
38. Bubniak, G. A.; Schreiner, W. H.; Mattoso, N.; Wypych, F.; Langmuir 2002, $18,5967$.

39. Schnitzler, D. C.; Meruvia, M. S.; Hummelgen, I. A.; Zarbin, A. J. G.; Chem. Mater. 2003, 15, 4658.

40. Huguenin, F.; Gambardella, M. T. D.; Torresi, R. M.; de Torresi, S. I.; Buttry, D. A.; J. Electrochem. Soc. 2000, 147, 2437.

41. Huguenin, F.; Torresi, R. M.; Buttry, D. A.; da Silva, J. E. P.; de Torresi, S. I. C.; Electrochim. Acta 2001, 46, 3555.

42. Ferreira, M.; Huguenin, F.; Zucolotto, V.; da Silva, J. E. P.; de Torresi, S. I. C.; Temperini, M. L. A.; Torresi, R. M.; Oliveira, O. N.; J. Phys. Chem. B 2003, 107, 8351.

43. Pohlmann, A. R.; Weiss, V.; Mertins, O.; da Silveira, N. P.; Guterres, S. S.; Eur. J. Pharm. Sci. 2002, 16, 305.

44. Corio, P.; Santos, P. S.; Brar, V. W.; Samsonidze, G. G.; Chou, S. G.; Dresselhaus, M. S.; Chem. Phys. Lett. 2003, 370, 675.

45. Corio, P.; Santos, A. P.; Santos, P. S.; Temperini, M. L. A.; Brar, V. W.; Pimenta, M. A.; Dresselhaus, M. S.; Chem. Phys. Lett. 2004, 383, 475.

46. Winnischofer, H.; Lima, S. D.; Araki, K.; Toma, H. E.; Anal. Chim. Acta 2003, 480, 97.

47. Dovidauskas, S.; Toma, H. E.; Araki, K.; Sacco, H. C.; Iamamoto, Y.; Inorg. Chim. Acta 2000, 305, 206

48. Kleverlaan, C. J.; Indelli, M. T.; Bignozzi, C. A.; Pavanin, L.; Scandola, F.; Hasselman, G. M.; Meyer, G. J.; J. Am. Chem. Soc. 2000, 122, 2840.

49. Argazzi, R.; Iha, N. Y. M.; Zabri, H.; Odobel, F.; Bignozzi, C. A.; Coord. Chem. Rev. 2004, 248, 1299.

50. Garcia, C. G.; de Lima, J. F.; Iha, N. Y. M.; Coord. Chem. Rev. 2000, 196, 219.

51. Azzellini, G. C.; Anais da Academia Brasileira de Ciencias 2000, 72, 33.

52. Lehn, J.-M.; Angew. Chem., Int. Ed. 1988, 27, 89.

53. Lehn, J.-M.; Supramolecular Chemistry: Concepts and Perspectives, VCH: New York, 1995.
54. Atwood, J. L.; Davies, J. E. D.; Macnicol, D. D., eds.; Comprehensive Supramolecular Chemistry; Pergamon Press: New York, 1996, Vol. 1-10.

55. Olenyuk, B.; Whiteford, J. A.; Fechtenkotter, A.; Stang, P. J.; Nature 1999, 398, 796.

56. Balzani, V.; Scandola, F.; Supramolecular Photochemistry; Ellis Horwood: Chichester, UK, 1991.

57. Balzani, V.; Scandola, F. Em ref. 2, Vol. 10, p. 687.

58. Bard, A. J.; Integrated Chemical Systems. A Chemical Approach to Nanotechnology, John Wiley \& Sons, Inc.: New York, 1994.

59. Araki, K.; Toma, H. E.; Supramolecular Porphyrins as Electrocatalysts, Springer: New York, 2006, Chapter 6.

60. Collman, J. P.; Anson, F. C.; Barnes, C. E.; Bencosme, C. S.; Geiger, T.; Evitt, E. R.; Kreh, R. P.; Meier, K.; Pettman, R. B.; J. Am. Chem. Soc. 1983, 105, 2694.

61. Collman, J. P.; Bencosme, C. S.; Durand, R. R.; Kreh, R. P.; Anson, F. C.; J. Am. Chem. Soc. 1983, 105, 2699.

62. Collman, J. P.; Denisevich, P.; Konai, Y.; Marrocco, M.; Koval, C.; Anson, F. C.; J. Am. Chem. Soc. 1980, 102, 6027.

63. Shi, C.; Anson, F. C.; J. Am. Chem. Soc. 1991, 113, 9564.

64. Shi, C. N.; Anson, F. C.; Electrochim. Acta 1994, 39, 1613.

65. Shi, C. N.; Anson, F. C.; Inorg. Chim. Acta 1994, 225, 215.

66. Shi, C. N.; Anson, F. C.; Inorg. Chem. 1995, 34, 4554.

67. Winnischofer, H.; Otake, V. Y.; Dovidauskas, S.; Nakamura, M.; Toma, H. E.; Araki, K.; Electrochim. Acta 2004, 49, 3711.

68. Toma, H. E.; Nikolaou, S.; J. Chem. Res. - S 2000, 326.

69. Toma, H. E.; Araki, K.; Silva, E. O.; Monatsh. Chem. 1998, 129, 975.

70. Collman, J. P.; Rapta, M.; Broring, M.; Raptova, L.; Schwenninger, R.; Boitrel, B.; Fu, L.; L'Her, M.; J. Am. Chem. Soc. 1999, 121, 1387.

71. Collman, J. P.; Schwenninger, R.; Rapta, M.; Broring, M.; Fu, L.; Chem. Commun. 1999, 137.

72. Gunter, M. J.; Turner, P.; Coord. Chem. Rev. 1991, 108, 115. 